\title{
Estimators for Logic Minimization and Implementation Selection of Finite State Machines
}

\author{
A.J.W.M. ten Berg \\ University of Twente \\ Faculty of Computer Science \\ P.O.Box 217, NL. 7500 AE Enschede, The Netherlands
}

\begin{abstract}
This paper considers two estimation problems which occur during the implementation design for a finit: state machine (FSM). The first is a precise estimation of the reduction of a progranmed logic aray implementation (PLA) for a FSM by logic minimization. The second concerns selection of implementation altematives based on such estimations. Estimations give the designer a quick overview of the impact of an optimization method for FSM implementation without running the actual time-consuming algorithms. The method uses curve-fitting on results found in literanure for logic minimization preceded by state-assignment. Our estimations correlate by 0.97 to those results. State-graph statistics can also be used for selection of the most profitable optimization from a set of alternatives. We tested selection between a counter based implementation, partial state coding, state-assignment and topological partitioning. The goal is selection $\mathrm{cf}$ the altemative which has the highest probability to deliver the largest minimization of the FSM. This selection method is also empirically verified by comparing its results with results obtained by running specific optimization algorithms on machines of the MCNC bencimark set.
\end{abstract}

\section{Introduction}

A considerable problem in synthesis is the selection of the minimal implementation alternative for Finite State Machines. Many implementation altematives are known [2][5] for specific hardware configurations. Each of these implementations has its specific optimization or minimization algorithms [2],[3],[12]. Thus, for a proper comparison of alternatives, the designer must execute all optimization algorithms. Unfortunately, this takes large computational efforts. Equal problems arise also in design 4janning [15], were design plans must be build. Quick estimations of optimizations can be of great help in such situations. Estimations improve the efficiency of the design process. One can identify two types of estimations for evaluation of design allernatives.

The first estimation type is the prediction of results of complex and therefore time-consuming optimizations. Such optimizations are usually bound to one implementation. An inportant example of such an optimization is the two-level logic mininization problem for Finite State Machines (FSM) implemented in a single PLA. However, only if the state-assignment problem [3] , [4] is soived, the minimal PLA is obtained. The state-assignment assigns binary codes to internal FSM states such that a two-level or a multi-level logic optimization has its maximal effect. In this case we consider only two-level logic ie. PLA implementations. The exact amount of minimization is not the most interesting to know. In most cases the relative minimization potential is far more useful for the designer. In other words, whether application of logic mirimization with state-assignment will reduce the machine size by $20 \%, 50 \%$ of maybe even $70 \%$.

The second estimation type concerns the selection probleza among implementation alternatives. For selection, not the accuracy of estimation for each individual altemative is important, but its selectivity. Therefore, such estimations must have a reasonable correlation with the resulis of their optimization algorithm and preferably a low conclation with the results of other optimizations. Selection estimations deliver the altemative with the highest probability of obaining the minimal implementation.

In order to test the estimators, we ran optimization algorithms for each alternative on the MCNC benchmark set of FSM's. This benchmark set is used widely for the comparison of state-assignment algorithms. The correlation coefficient between estimations and agorithm results provides us an empirical verification. The set of alternatives contains a counter, partial state code generation, state-assignment and topological paritioning. Section 2 discusses the curve-fit estimation method for 
state-assignment. Section 3 explains the optimizations for each altemative and also the statisxical measures used for the selection.

\section{State-assignment result estimation}

What we try to estimate is the maximal reduction of a PLA implementation of a FSM by logic minimization. This problem includes the state-assignment problem, which must be solved optimal in order to obtain the maximal profit out of logic minimization. The state-assignment problem is very complex and as problem hard to estimate. The FSM structure however is much simpler and therefore we can estimate the maximal profit of logic minimization for it.

In other engineering sciences as foi exarpple in chemistry, curve-fitting is usually applied to model complex physical processes. In chemistry, the behaviour of reactors is modelled by volume, pressure and temperature parameters, which are very global compared to the actual molecular reactions. Then. curve-fit methods can supply understanding and prediction of the macro-molecular behaviour of the complete reactor. Therefore, we investigate a curve-fitting method for the estimation of two level logic minimization. Analogue to the example we have to identify global FSM parameters as input for the estimation polynomial.

The curve-fit methot delivers the coefficients $C$ for a polynomial in the form of (1). The input data for the curve-fit are the results from NOVA [9]. NOVA performs near-optimal state-assignments for PLA implementations, and it obtains its minimization results with ESPRESSO [11], which is a widely known two level logic minimizer. The number of coefficients of the polynomial minus one is the order of the polynemial. We applied mathematical APL programs for regression analysis [1] to compute the coefficients $\mathbf{C}$ of the polynomial curve. These programs apply a least square's method for this computation.

Polynomial of order 4 :

$\operatorname{POL} Y(x)=\quad \mathrm{C} 1 . x * 4+\mathrm{C} 2 \mathrm{x}^{* * 3}+\mathrm{C} 3 \cdot \mathrm{x}^{* * 2} 2+$ $\mathrm{C} 4 \mathrm{x}+\mathrm{C5}$

The first problem is to determine the parameter(s) $x$ of the FSM that give a curve close to the NOVA/ESPRESSO results. The second problem is the order of the polynomial. This order must be small compared to the set of data from which the polynomial is derived. NOVA [9] provides restitis for 25 machines from the MCINC benchnark set (table 1). For a proper carve, the polynomial order must be small compared to the number of samples. Therefore we use a polynomial of a third order, which is small enough.

\subsection{Finite State Machine parameters.}

Next, we identify some curve farameters, for which we need first some FSM definitions. Cardinality of a set of elements is denoted by \#. For example: \#I is the number of symbols in the input alphabet. A binary coded symbol is called a word. A Finite State Machine is defined by the quintuplet $\mathrm{FSM}=\langle\mathrm{I}, \mathrm{O}, \mathrm{S}$, OUT, TRS > given by :

$\begin{array}{ll}\mathbf{I}=\left\{\mathrm{i}_{1} . \mathrm{i}_{\mathrm{k}}\right\} & \begin{array}{l}\text { Input alphabet where } \mathrm{i}_{\mathrm{x}} \text { is a binary } \\ \text { coded symbol }\end{array} \\ \mathbf{O}=\left\{01 . . \mathrm{O}_{\mathrm{m}}\right) & \begin{array}{l}\text { Output alphabet where ox is a } \\ \text { binury coded symbol }\end{array} \\ \mathbf{S}=\left\{\mathrm{s}_{1} . . \mathrm{s}_{\mathrm{v}}\right\} & \begin{array}{l}\text { State alphabet, where } \mathrm{s}_{\mathbf{x}} \text { is } \\ \text { symbolic. }\end{array}\end{array}$

$0:=\operatorname{OUT}(\mathrm{s}, \mathrm{i}) \quad$ Mealy Output function

$0:=\operatorname{OUT}(s) \quad$ Moore Output function

$s^{*}:=\operatorname{TRS}(s, i) \quad$ Transition function

A second specification of a FSM is given by a set PTS $=\{\mathrm{Pt}]$..pin $\}$ of four tuples <input word, state, next state, output word $>$ denoted as $p t=<i, s, s^{\prime}, 0>$ which are called productterms. The FSM's in the benchmark set are all of the Mealy type. Now we can write the estimation of the minimized number of productterms \#PTS ${ }_{\min }$ in (2).

$$
\text { \#PTS } \min =\operatorname{POLY}(x) * \text { \#PTS }
$$

We select various parameters, and combinations of them, for the $\mathrm{x}$ in (2) and tested their quality. First we compute the polynomial coefficients. Then, the estimations of \#PTS $_{\text {min }}$ are compared with the NOVA/ESPRESSO [9] results. This comparison includes the correlation coefficient and the average difference between the algorithn minimization and the estimated minimization APTS min. Examination of the FSM structure in relation with the NOVA [9] results shows two important FSM parameters. The first paraneter is the number of productierms per state \#PTS/\#S. One expects that for logic minimization, a high number of producterms for a stats causes a relatively high minimization potencial. Because, if more productterms share the stame state code then Jarger cubes can be expected. A second parameter is the number of output variables \#O. Roughly, the higher this number, the more difficult it becomes to combine productterms. Thus the number of output variables contributes in a reverse way to minimization. Therefore we use this parameter inverted $(1 / \# 0)$ when we combine it with other parameters as \#PTS/\#S. Furthermore, the number of inpu: variables \#I may be interesting. Although \#I is mostly related to \#PTS/\#S and therefore only a limited effect can be expected from its use. 
A combination of more FSM parameters in $x$ requires a weighting mechanism. This to make sure that value ranges of parameters become overlapping. Thus, we presume an equal importance of both parameters. The value range of the \#PTS/\#S parameter is roughly from 1 to 10 . The $1 /$ / 0 parameter is always between 0 and 1 . Therefore this last parameter is multiplied with a factor 10. Another altemative would be the use of complex curve fitting, which can obtain a polynomial for sets of parameters. However, the results of parameter balancing are quite good in this case, which makes complex curve-fitting superfluous here.

\begin{tabular}{|l|c|c|c|c|} 
& $\# 1$ & $\# 0$ & \#PTS & \#S \\
\hline bbara & 4 & 2 & 60 & 10 \\
bbsse & 7 & 7 & 56 & 16 \\
bbtas & 2 & 2 & 24 & 6 \\
beect & 3 & 4 & 28 & 7 \\
cse & 7 & 7 & 91 & 16 \\
dk14 & 3 & 5 & 56 & 7 \\
dk15 & 3 & 5 & 32 & 4 \\
dk16 & 2 & 3 & 108 & 27 \\
dk17 & 2 & 3 & 32 & 8 \\
dk27 & 1 & 2 & 14 & 7 \\
dk512 & 1 & 3 & 30 & 15 \\
donil & 2 & 1 & 96 & 24 \\
ex1 & 9 & 19 & 138 & 20 \\
ex2 & 2 & 2 & 72 & 19 \\
ex3 & 2 & 2 & 36 & 10 \\
ex5 & 2 & 2 & 32 & 9 \\
ex6 & 5 & 8 & 34 & 8 \\
keyb & 7 & 2 & 170 & 19 \\
plan & 7 & 19 & 115 & 48 \\
s1 & 8 & 6 & 107 & 20 \\
sand & 11 & 9 & 184 & 32 \\
sct & 27 & 56 & 166 & 121 \\
shift & 1 & 1 & 15 & 8 \\
styr & 9 & 10 & 166 & 30 \\
tra11 & 2 & 1 & 25 & 11 \\
\hline
\end{tabular}

Table 1. Data of MCNC FSM set.

Table 1 gives the MCNC FSM data and Table 2 shows a comparison of FSM pararaters and some combinations of them. Clearly $x=(10 / \# O)+(\# P T S / \# S)$ delivers the best result with a correlation coefficient of 0.97 with the NOVA results and an average difference of $6.1 \%$ in $A P T S_{\min }$

\begin{tabular}{|l|c|l|}
\hline parameter : $x$ & Corr.Coeft. & Av.Difl.\% \\
\hline$\# P T S / \# S$ & 0.94 & 10.3 \\
$\# O$ & 0.94 & 9.5 \\
$(10 / \# O)+(\# P T S / \# S)$ & 0.97 & 6.1 \\
$\# 1+(10 / \# O)+(\# P T S / \# S)$ & 0.92 & 11.2 \\
$\# 1$ & 0.92 & 11.2 \\
$\# 1+(10 / \# O)$ & 0.93 & 10.4 \\
$\# 1+(\# P T S / \# S)$ & 0.92 & 12.1 \\
\hline
\end{tabular}

Table 2. Estimations for several FSM parameters compared. compared with the NOVA results. Then, Table 3 gives the coefficients of the polynomial for $x=(10 / \# 0)+(H P T S / H S)$. Table 4 shows in the second column the productterms after minimization \#PTS $\mathrm{min}$, computed with the polynomial. The first column shows the number of productems obtained by NOVAVESPRESSO. Table 5 gives the percentile differences between estimation and algorithrn. related to the original \#PTS of the machines.

\begin{tabular}{|c|c|}
\hline coetficient & order \\
\hline $3.23 \mathrm{E}-4$ & $x^{4+3}$ \\
$9.60 \mathrm{E}-3$ & $x^{4+2}$ \\
-0.13 & $x^{* m 1}$ \\
1.03 & 1 \\
\hline
\end{tabular}

Table 3. Coefficients of estimation polynomial.

These tables show the remarkahle precision of the estimations. Certainly, because the estimations are based on only two parameters of the FSM and a third order polynomial. This gives sufficient confidence in the usefulness of this type of estimations for prediction of the impact of logic minimization including state-assignment. The two largest exceptions are machines $s 1$ and exl. We analysed these machines into further detail but did not identify a clear cause. Further research concems estimations for olher optimization algorithms used in

\begin{tabular}{|l|c|c|}
\cline { 2 - 3 } \multicolumn{1}{c|}{} & \multicolumn{2}{c|}{ \#Fis } \\
\cline { 2 - 3 } bbara & NOVA & astim. \\
bbsse & 24 & 23 \\
bbias & 8 & 34 \\
beect & 11 & 11 \\
cse & 44 & 15 \\
dk14 & 22 & 45 \\
dk15 & 16 & 13 \\
dk16 & 49 & 53 \\
dk17 & 17 & 16 \\
dk27 & 8 & 7 \\
dk512 & 17 & 18 \\
donil & 23 & 25 \\
ex1 & 40 & 67 \\
ox2 & 29 & 32 \\
ex3 & 17 & 17 \\
ox5 & 15 & 15 \\
ox6 & 23 & 20 \\
keyb & 47 & 44 \\
plan & 87 & 86 \\
st & 80 & 54 \\
sand & 69 & 93 \\
sef & 138 & 140 \\
shitt & 4 & 6 \\
styr & 89 & 86 \\
trat1 & 9 & 8 \\
\hline & & \\
\hline
\end{tabular}

Table 4. NOVA results with estimations.

\begin{tabular}{|c|c|}
\hline & \%Dilt. \\
\hline $6 \times 5$ & 0.0 \\
\hline mark1 & 0.0 \\
\hline tral1 & 0.0 \\
\hline styr & 1.2 \\
\hline bbara & 1.6 \\
\hline koyb & 1.7 \\
\hline dk14 & -1.8 \\
\hline donfli & -2.1 \\
\hline sand & -2.2 \\
\hline $\operatorname{css} \theta$ & -2.2 \\
\hline set & -2.5 \\
\hline ptan & 2.6 \\
\hline $6 \times 3$ & 2.7 \\
\hline ck17 & 3.1 \\
\hline C.5512 & -3.4 \\
\hline ck16 & -3.8 \\
\hline ex2 & -5.6 \\
\hline dk2? & 7.1 \\
\hline $0 \times 6$ & 8.8 \\
\hline dk15 & 9.3 \\
\hline bbsse & -12.5 \\
\hline bbtas & -12.5 \\
\hline shift & .12 .5 \\
\hline berect & $\cdot 14.3$ \\
\hline ex 1 & -19.6 \\
\hline s1 & 24.2 \\
\hline
\end{tabular}

Table 5. Percentile differences. 
microarchiteciure synthesis. The next section discusses a different type of estimations that can select a microarchitecture from several altematives. This requires a selective character instead of a bigh precision.

\section{Implementation alfermative selection by estimations.}

The second type of estimation problem is the selection among implementation altematives. In most cases, the designer has to choose from a set of alternative implementations. Unfortunately, each implementation usually requires its own specific optimization. Therefore a proper comparison between alternatives requires execution of all the optimization algorithms. Estimations can provide the designer quickly with information on the altematives. Then, the designer can deduct which of the alternatives are most promising for a more detailed comparison. We implemented a set of implementation alternatives and computed results of their optimization algorithms for the machines in the MCNC benchmark set. Next is the FSM analysis which is the base for the estimations. The problem is to identify those FSM parameters that have a selective character. Therefore, these parameters must provide a high correlation between the estimation and its optimization algorithm results and a low correlation with the other afiematives.

We focus mainly on the transition function in the FSM, because quite a number of different altematives is available to implement it. First we explain each of the altematives shortly with theirs related optimization problem. Some optimization algorithms originate from literature and some where necessarily self-developed. The alkematives set contains a counier implementation, partial state coding, state assignment and topological partitioning. These are all quite common to FSM's and not restricted to very specific types of FSM's as other more dedicated optimizations [7],[8] are.

\subsection{A counter implementation.}

Algorithms for optimal embedding of a counter in the transition function of . FSM are studied extensively in [2]. The counter implements the cotuntable transitions. This makes these transitions redundant in the transition PLA and therefore removable. The PLA generates the signal to. cownter activation by default in case an input pattern does not match any of its productuerms. The counter must be loadable to accept codes generated by the PLA in case of non-couniable transitions. The counter connects to the transition function oupur, thus it affects only transition codes.
The optimization coupled to this implementation is $\mathrm{L}$.. coding of transitions such that the countable transitions cover the highest possible number of producterms (PTS). Thus, the algorithm must identify a collection of transition chains that cover a miximum number of producterms. Such an algorithm is given in [2]. That algorithm handles only Moore type machines and incorporates also other constraints for the code assignment which make it less suitable for our purpose. Because the MCNC benchmark set contains Mealy machines, we developed a different algorithm better suited to Mealy machines.

Countable transition chain $\mathrm{Cr}_{\mathbf{i}}$ :

$$
\begin{aligned}
& C t_{i}=t_{s} 1 . . t r s k \\
& 1<=i<=k(3)
\end{aligned}
$$

The total number of productterms implemented by a counter is then :

$$
\begin{aligned}
& \text { n } \\
& \text { PTSC }=\sum \# p t\left(C_{i}\right) \text { where } n \text { is the number of } \\
& \text { countable chains } \\
& \mathrm{i}=1
\end{aligned}
$$

The objective of the algorithm is to code TRS such that PTSC is maximal. Therefore all transitions get a weight assigned representing their number of productterms : $w i=$ fpt(trsi). The algorithm removes first all transitions of which the start state is equal to the next state, because transition cycles are not countable. It sorts the transitions to decreasing weights. In the next phase, the algorithm builds chains of transitions. It starts with the first unmarked transition and scans the list for follow-up transitions. Thus, the transitions with the highest weight are selected first. The algorithm proceeds with this until it encounters a cycle, or it finds no follow-up transition. Furthermore, it marks only the state which is at the head of a chain. Through this way of state marking a state may occur in more than one chain. It occurs however only once as head of a chain. This redundancy avoids tixial optima. The algorithm genertes new chains as long as there exist unmarked states that have unmarked successors. Next, the chains are sorted according their cumulative weights, again in a decreasing order. To retrieve the final collecrion of countable chains, the algorithm takes chains from the head of the list. Chains that contain states which do also occur in previously selected chains are rejected. The algorithm codes the states according the chains selected. This algorithm iterates until no improvenent occurs in the PTSC. Table 6 shows the results for this algorithm on the MCNC FSM benchmark set. Column d.Cntrl shows the number of countable productterms: before optimization, d.Cntr2 after optimization. These results are also in table 7 , column \#PTS_c. 


\begin{tabular}{|c|c|c|c|}
\hline & \#PTS & d.Cntrt & d.Cnis \\
\hline bbara & 60 & 11 & 15 \\
\hline bbsse & 55 & 8 & 11 \\
\hline bbtas & 24 & 10 & 11 \\
\hline beect & 28 & 4 & 7 \\
\hline $\operatorname{cs\theta } \theta$ & 91 & 4 & 14 \\
\hline dk14 & 56 & 5 & 7 \\
\hline dk15 & 32 & 3 & $\mathbf{5}$ \\
\hline dk16 & 108 & 11 & 26 \\
\hline dk17 & 32 & 7 & 9 \\
\hline dk27 & 14 & 3 & 5 \\
\hline dk512 & 30 & 7 & 12 \\
\hline donfil & 96 & 7 & 13 \\
\hline $0 \times 1$ & 138 & 3 & 21 \\
\hline $0 \times 2$ & 72 & 6 & 18 \\
\hline $0 \times 3$ & 36 & 3 & 9 \\
\hline $9 \times 4$ & 21 & 11 & 13 \\
\hline $0 \times 5$ & 32 & 4 & 8 \\
\hline ex6 & 34 & 3 & 9 \\
\hline$e \times 7$ & 36 & 6 & 10 \\
\hline keyo & 170 & 12 & 44 \\
\hline lion & 11 & 3 & 3 \\
\hline liong & 25 & 6 & 6 \\
\hline plan & 115 & 6 & 50 \\
\hline 31 & 107 & 6 & 23 \\
\hline sand & 184 & 2 & 82 \\
\hline sct & 156 & 31 & 120 \\
\hline shilt & 16 & 4 & 8 \\
\hline styr & 166 & B & 56 \\
\hline lav & 49 & 2 & 23 \\
\hline trao4 & 14 & 5 & 6 \\
\hline tral1 & 25 & 1 & 8 \\
\hline
\end{tabular}

Table 6. Improvement in countable productterms.

\subsection{Partial state coding}

Partial state coding is not a contmon optimization type in controlpath synthesis systems. However, many manual designs of processor controlpaths [10] use this method to implement state transitions. It reduces the number of producterms in case several states have identical transition structures and active input variable sets. The transiticn function generates just a part of the next address (state code), often only the two least significant bits (LSB). The

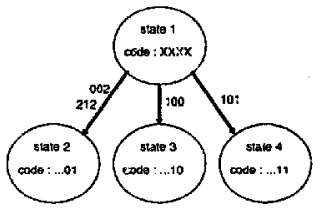

Figure 1. Example of partial coding of sucessor states. output function generates the other, the most significant, bits (MSB) of the next address. By this, these MSB bits must be identical in all next states of a certain state. This state code generation is feasible because states usually have only a small number of next states as shown in figure 1. That makes it possible to code the local selection of the next state in a few bits. Then the MSB bits of the state code can be equal among all next states of a state. That makes it possible to generate the MSB part with the output function. In case several states have identical sets of input vectors and transitions, they cian share a set of producterms in the transition PLA. This reduces the number of productierms and output variables in the transition PLA. Note the cost of additional output variables for the MSB bits in the output function. The optimization problem is to find the maximal number of compatible transition sets. As stated before, transition sets compatible in case all inputs are identical and the sets of next states contain equai subsets of states. Furthermore these sets may contain each state only once to prevent muztiple codes for a single state. In case not all states are partially codeable, the transition function decomposes into two PLA's, of which one generates the usual conplete next state code for all state transitions that cannot be implemented by partial state coding.
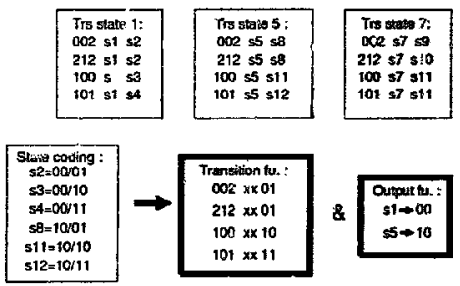

Figure 2. Exarnple of productterm reduction by partial codes

The example of figure 2 shows a reduction in productrerms. In this example, the transition set of state 7 is not identical with the other two sets because its next state structure has a different pattern. Both the input word set and the transitions have to be identical, as is the case for states 1 and 5. After state coding, the transitions of states 1 and 5 can be merged. However, the transitions from state 7 cannot be implemented in the same PLA as the merged set of states 1 and 5 . The output function generates the static code part, in case of state 1 it generates 00 , of state $5: 10$, and for state 7 this code is don't care : 22. 


\section{Optimization}

The next algorithm detects the number of redundant productterms in a FSM for a dbits wide LSB part of the state code. Firsts it removes all transitions starting from states that have more than $2^{* *} d$ dbits of next states. Then it generates maximal clusters of states with identical input vectors. From thase clusters the algorithm makes new ones that contain the next state collections in a matrix form with one row of nexi states for each state. For partial state coding, a clusicr should contain only unique states. Therefore the algorithm searches for a maximal unique sub-matrix. It discards non-unique states from a state/transition matrix one by one until a sub-matrix with unique states remains. The reduction in transitions is then obtained by counting the number of states in the unique submatrix minus one row, because one row is actually implemented. From this follows also the number of redundant productterms. In table 7, column \#PTS_a gives the Ieduction of productterms of the transition function. This methoi is suited best for control functions with a few transitions per state and disjoint next-state sets.

\begin{tabular}{|c|c|c|c|c|c|c|}
\hline & \#PTS & *S & \#PTS_a & \#PTS_c & \#PTE_s & \#PTS_t \\
\hline bbera & 60 & 10 & 6 & 15 & 36 & 0 \\
\hline bbsse & 56 & 16 & 3 & 11 & 29 & 7 \\
\hline bboas & 24 & 6 & 8 & 11 & 16 & 0 \\
\hline beect & 28 & 7 & 0 & 7 & 17 & 0 \\
\hline $\operatorname{cse}$ & 91 & 16 & 0 & 14 & 47 & 6 \\
\hline dK 14 & 56 & 7 & 0 & 7 & 34 & o \\
\hline dkt5 & 32 & 4 & 0 & 5 & 16 & 0 \\
\hline హk16 & 108 & 27 & 58 & 26 & 59 & $n$ \\
\hline $\mathrm{dk} 17$ & 32 & 8 & 8 & 9 & 15 & 0 \\
\hline$d k 27$ & 14 & 7 & 2 & 5 & 6 & 0 \\
\hline dk512 & 30 & 15 & 16 & 12 & 13 & 0 \\
\hline donit & 96 & 24 & 8 & 13 & 73 & D \\
\hline ex: & 138 & 20 & 2 & 21 & 98 & 31 \\
\hline ex2 & 72 & 19 & 28 & 18 & 44 & 0 \\
\hline ex3 & 36 & 10 & 12 & 9 & 19 & 0 \\
\hline ex4 & 21 & 19 & 2 & 13 & . & 1 \\
\hline $6 \times 5$ & 32 & 9 & 8 & 8 & 17 & i \\
\hline $\operatorname{ex5}$ & 34 & B & 0 & 9 & 11 & 0 \\
\hline$e \times 7$ & 36 & 10 & 16 & 10 & . & $v$ \\
\hline keyb & 170 & 19 & 0 & 44 & 123 & f \\
\hline lion & 11 & 4 & 3 & 3 & . & 0 \\
\hline $\operatorname{tin} 9$ & 25 & 9 & 0 & 6 & . & 0 \\
\hline plan & $1 \pm 5$ & 48 & 34 & 50 & 28 & 18 \\
\hline 51 & 107 & 20 & 3 & 23 & 27 & 5 \\
\hline sand & 184 & 32 & 34 & 82 & 95 & 36 \\
\hline$s c t$ & 166 & 121 & 10 & 120 & 28 & 78 \\
\hline shift & 16 & $B$ & 14 & 0 & 12 & 0 \\
\hline styr & 166 & 30 & 0 & 56 & 77 & 26 \\
\hline tav & 49 & 4 & 0 & 23 & . & 0 \\
\hline 15:04 & 14 & 4 & 0 & 6 & - & 0 \\
\hline trat1 & 25 & 11 & D & $B$ & 16 & 0 \\
\hline & t & & $\mathrm{m} 1$ & & & \\
\hline
\end{tabular}

Table 7. Algorithm results : producterm reduction.
Microprogramming methods [10] use that knowledge extensively to generate the controlpath function which can be implemented efficiently with this technique.

\subsection{State Assignment.}

As is clear from section 2, logic minimization with state assignment reduce also the number of productterms of a PLA implementation. Therefore, we consider it as another design altemative. Table 7 shows the results of NOVA/ESPRESSO [9] as \#PTS - \#PTS $\min$ in column \#PTS_s. Unfortunately NOVA does not supply data for all machines in the MCNC set

\subsection{Topological Partitioning.}

This problem concerns the division of a single PLA into several smailer PLA's such that the AND- and OR-planes of the PLA's become densely used, which reduces the total silicon area occupied. Several algorithms we known for this problem [12], [13], [14]. We selected the algorithm of Hennessy [13] and extended it to FSM's by viewing all productterms in a state as an entity, which simplifies implementation and reduces computational complexity. For more details on the algorithm we refer to [14]. One variant of this topological partitioning algorithm minimizes the total area of the PLA's, if possible. It accounts also for input/output buffers, power and ground overhead. The last column of table 7, called \#PTS_t, shows the converted results. The original results are in terms area reduction. These were converted to numbers of productterms which take the equal amount of area, to make topological partitioning results comparable with the results of the other algorithms.

\subsection{FSM analysis.}

This section explains how the estimations for selection are made. Both the state-graph structure and $I$ and $O$ word densities are analysed. The most obvious analysis concerns the division of numbers of transitions per state. We started with simple estimations based on observations and computed the correlation coefficients. Then we tried to improve these correlations by exploring closely related alternatives. The tables do not show these alternatives for sake of clarity. We discuss only the final estimations in the remainder of this section. Table 8 shows the reduction of productterms \#PTS-\#PTS $\min$ for each estimation.

The column 'subsetl' of table 8 estimates countable transitions. It estimates the average number of countable transitions as the number of states times the average number of productterms for a transition (\#S*\#PTS/\#TRS). We know that just one countable transition can occur for 
each state and also the average number of productterms in a transition. This gives the average expectation of the number of productterms to be saved.

\begin{tabular}{|c|c|c|c|c|}
\hline & subset1 & subset2 & subset3 & subset4 \\
\hline bbara & 16 & 0 & 60 & 1 \\
\hline bbsse & 21 & 12 & 38 & 12 \\
\hline bbtas & 12 & 24 & 24 & 0 \\
\hline beect & $\mathbf{B}$ & 28 & 28 & 0 \\
\hline $\operatorname{cs} \theta$ & 26 & a & 91 & 7 \\
\hline dk14 & 14 & 0 & 56 & 1 \\
\hline dk15 & 10 & 0 & 32 & 0 \\
\hline$d \mathbf{k} 16$ & 28 & 108 & 100 & 2 \\
\hline dk17 & 11 & 32 & 32 & 0 \\
\hline dk27 & 11 & 8 & 0 & 0 \\
\hline dk512 & 15 & 30 & 0 & 0 \\
\hline donfl & 24 & 96 & 96 & 1 \\
\hline $\mathrm{ex} 1$ & 37 & 12 & 130 & 24 \\
\hline $\operatorname{ex} 2$ & 24 & 72 & 72 & 1 \\
\hline $\operatorname{ex} 3$ & 12 & 36 & 36 & 1 \\
\hline$e \times 4$ & 16 & 10 & 0 & 7 \\
\hline ex5 & 11 & 32 & 32 & 0 \\
\hline ex6 & B & 16 & 31 & 3 \\
\hline$e \times 7$ & 13 & 36 & 36 & 1 \\
\hline kayb & 70 & 12 & 158 & 19 \\
\hline lín & 4 & 2 & 0 & 0 \\
\hline liong & 9 & 4 & D & 0 \\
\hline plan & 77 & 46 & 52 & 52 \\
\hline s1 & 26 & 20 & 86 & 22 \\
\hline sand & 65 & 44 & 136 & 69 \\
\hline sct & 133 & 32 & 34 & 133 \\
\hline shift & $\mathbf{B}$ & 16 & 0 & 0 \\
\hline styr & 54 & 4 & 153 & 37 \\
\hline lav & 49 & 0 & 49 & 0 \\
\hline ira04 & 7 & 8 & $\mathrm{~B}$ & 0 \\
\hline 1ra11 & 11 & 16 & 0 & 0 \\
\hline
\end{tabular}

Table 8. Productern reduction estimations.

Column 'subset2' is for partial state coding. The number of productterms per state relates strongly to the number of code bits generated by the transition function. We choose for two bits. This allows at most four next states in a state. Therefore we count all productterms of states which have exactly two or four productterms (\# $\{\mathrm{pt}$ leaving $s \mid=2,4)$.

Next, column 'subset3' is for state assignment. A high number of productterms per state gives on average better logic minimization results. We counted the total number of productterms of states with four or more productterms (\#) lpt leaving s) $>=4$ ). After some experiments, this number gave the highest correlation with state-assignment results.

The last column 'subset4' of table 8 shows the productterm reduction equivalent of the area saved with topological partirioning. This equivalent gives compatibility with the other subsets, which is needed for a correct determination of the correlation coefficients.

This number of productterms is derived from the average number of inputoutput variables active per state. This number lies between 0 and 1 . The lower this number the higher the possible area savings by topological partitioning. The next formula was derived experimentally to correspond with the optimization results from 3.4. *.pTS $=1 /($ \#Is/\#S + C* \#Os/\#S) were \#Is is the totsl of active input variables counted over all states. \#Os is the ompar variable equivalent of this. $\mathrm{C}=50$ is an experimentally derived constant to balance both parameters.

\subsection{Results.}

Table 9 lists the computed correlation coefficients between the estimated reduction in \#PTS of table 8 and the algorithm results in table 7 . Table 9 shows quite bigh correlations on the main diagonal and reasonable low correlation coefficients with other optimization estin.ations. However, there is one exception, which is the correlation between counter and topological partitioning. Both the counter and topological partitioning estimations correlate highly with each others algorithm results.

\begin{tabular}{|l|c|c|c|c|}
\hline opt.method & subseti & subset2 & subset3 & subset4 \\
\hline counter & 0.95 & 0.17 & 0.46 & 0.95 \\
part.address & 0.22 & 0.78 & 0.24 & 0.18 \\
stalb-assign. & 0.45 & 0.18 & 0.93 & 0.27 \\
topol.partit. & 0.86 & 0.03 & 0.34 & 0.97 \\
\hline
\end{tabular}

Table 9. Correlation coefficients of estimations.

One can give two explanations for this. The first is that we measure the wrong parameter for both estimations. But. because the subsets $I$ and 4 are based one totally different parameters which are independent by nature, this explanation is not founded. The second explanation is that both optimizations are highly correlated for the FSM's in this benchmark set. To check this assumption, we computed also all the correlations between the results of the optimization algorithms. Table 10 lists them. All coefficients on the main diagonal are one, as expected. Furthermore, this table shows that the second explanation is correct, because the counter and topological partitioning algorithm results are here also highly correlated. In fact, the coefficients in table 9 resemble those in table 10 quite closely, which shows that our estimations for altemative selection are quite reliable.

\begin{tabular}{|l|c|c|c|c|}
\hline opt.mothnd & counter & part.addr & stalo-ass. & topol.pant \\
\hline counler & 1.00 & 0.28 & 0.41 & 0.91 \\
part.address & 0.28 & 1.00 & 0.15 & 0.10 \\
state-assign. & 2.41 & 0.15 & 1.00 & 0.32 \\
topol.panit. & 0.91 & 0.10 & 0.32 & 1.00 \\
\hline
\end{tabular}

Table 10. Correlations between optimization resulis. 
How must we interpret FSM estimations on alternatives with regard to these correlations?. In case the estimations for a FSM result in just one high estimation number, the selection is evident. When equal or nearly equal estimation figures occur for altematives, one selects the altemative which has the higher correlation coefficient in table 9 , because the correlation coefficient expresses the probability to be the best altemative. But, if the FSM has equal estimation figures for subsets 1 and 4 . no selection can be made based the correlation coefficients. Thus, only in this case one has to run both optimization algorithms. It is likely that both alternatives have about equal minimization potentials. Concluding, for most cases our estimations give a clear indication of the probably best alternative, which helps the designer to choose among altemative implementations.

\section{Cerducions.}

For the estimation of logic minimization including state-assignment, we conclude from the correlation coefficient of 0.97 with an average size difference of $6 \%$ compared to the algorithm results, that it is reliable enough to apply it in synthesis decisions. It gives a good expectation of what size reductions are fcasible with logic minimization for a FSM without the need for extensive computations. Although the high state-assignment problem complexiry, the FSM structure is less complex, which explains the high quality of our results.

The selection estimators, also based on FSM statistics, show correlation coefficients around 0.9 and cross-correlation coefficients that are typically below 0.4 . Only the highly correlated results of two optimizations in the altematives set distort these results partially. On average, these correlations help to retrieve information on whict design alternatives are the most suitable for a certin FSM. This selection method saves detailed computations for all optimizations for the alternatives. This makes this type of estimation methods also suitable for planning based design systems [15]. At this moment we extend our research on curve-fitting to other implementation techniques as multi-level logic implementations. Also, on the ficld of implementation selection, research for more elaborated sets of alternative implementations is carried out.

\section{References}

11] Thomson, N., APL Programs for the Mathematics Classroom., (Springer Verlag, New York, 1989).
[2] Amann, R., Algorithmische entwurfsverfahren fuer kombinierte pla/rom-steuerwerke unter verwendung van zaehlern, Dissertation, (VDI Verlag, Duesseldorf, 1987).

[3] DeMicheli, G., Optimal State Assignment for Finite State Machines, IEEE Trans. Comp. Aided Design, vol. CAD-4, (1985) pp. 269-284.

[4] Devadas, S. and Newton, R., Exact Algorithms for Output Encoding, State Assignment, and Four-Level Boolean Minimization, IEEE Trans. on CAD. Vol.10. No.1. January 1991, pp. 13-27.

[5] Obrebska, M., Efficiency and Performance Comparison of Different Design Methodologies for Control Parts of Microprocessors, Microprocessing and Microprogramming 10, (1982) pp.163-178.

[6] Tredennick, N., The "Cultures" of Microprogramming, Proc. of the 15th annual workshop on Microprogramming- Micro-15, 1982 , pp. 79-83.

(7) Paulin, P.G., Horizontal Partitioning of PLA-based Finite State Machines, Proc. of the 26th ACM/IEEE Design Automation Conference, 1989, pp. 333-338.

[8] Tarroux, G. et. al. Optimization of Micro-Controllers by Partitioning, Proc. EDAC 1991, Amsterdam, Feb, 1991, pp. 368-373.

(9) Villa, T. and Sangiovanni-Vincentelli A., NOVA; Staic Assignment of Finite State Machines for Optimal Two-Level logic implementation, IEEE Trans. on CAD, vol. 9 no. 9. sept 1990, pp. 905-924.

[10] Husson, S.S., Microprogrammin Principles and Practices, Prentice Hall, 1970.

[11] Brayton, R.K., et. al., Logic Minimization Algorithms for VLSI Synthesis, Kluwer Academic Publ, 1984.

[12] De Micheli, G. and Santomauro, M., "Smile ; A Computer Program for partitioning of programmed logic arrays", Computer Aided design, vol 15 No. 2, March 1983, pp. 89-97.

[13] Hennessy,J,, "Partitioning Programmable Logic Arrays Summary", IEEE Proc. Int. Conf. on Computer Aided Design, IEEE 1983, pp. 180-181.

[14] ten Berg, A.J.W.M., "Floorplan Optimized Topological Partitioning of Programmed Logic Arrays" accepted for publication at the WG 10.5 IFIP Workshop on Synthesis, Generation and Portability of Library Blocks for ASIC design. Grenoble, March 1992

[15] Knapp, D.W. and Parker, A.C., "The ADAM Design Planning Engine", IEEE Trans, on Comp. Aided Des., Vol. 10, No. 7, July 1991, pp. 829 - 345. 